\title{
Congestion management with aggregated delivery of flexibility using distributed energy resources
}

\author{
Calum Edmunds ${ }^{1, *}$,Ian Elders ${ }^{1}$, Stuart Galloway ${ }^{1}$, Bruce Stephen ${ }^{1}$, Andrey Postnikov ${ }^{2}$ \\ Liz Varga ${ }^{2}$, Yukun $\mathrm{Hu}^{2}$, Timoleon Kipouros ${ }^{3}$ \\ ${ }^{1}$ Dept. of Electronic and Electrical Engineering, Univeristy of Strathclyde, Scotland \\ ${ }^{2}$ Dept. of Civil, Environmental \& Geomatic Engineering, University College London, UK \\ ${ }^{3}$ Engineering Design Centre, Cambridge University, UK \\ *Corresponding author, email: calum.edmunds@strath.ac.uk
}

\begin{abstract}
Increasing penetrations of small scale electricity generation and storage technologies are making an important contribution to the decentralisation and decarbonisation of power system control and operation. Although not currently realised, coordination of local distributed energy resources (DERs) and a greater degree of demand flexibility through digital aggregation, offer the potential to lower the cost of energy at source and to enable remuneration for consumer participation, addressing the rising costs of energy supply, which impacts strongly on all consumers. Methods are required to manage potential distribution network constraints caused by flexible DERs, as well as for determining the risk to delivery of flexibility from these DERs for aggregators. A heuristic network flexibility dispatch methodology is proposed, which can be used to calculate the probability of constraints, and any required adjustments of flexible agent positions to resolve them, at half hourly resolution. The aggregator can use this methodology to manage their portfolio risk, while a distribution system operator can estimate required flexibility to manage constraints down to low voltage level.
\end{abstract}

Index Terms - aggregator, multi-agent, optimisation, network constraints, DERs, flexibility, DSO

\section{INTRODUCTION}

Low Carbon Technologies, particularly heat pumps, rooftop photo voltaic (PV) and electric vehicles (EV) are being connected at low voltage level in increasing penetrations globally. Traditional market arrangements rely on centralised models for managing the energy system, and the greatest barrier to end user participation in energy system operation is the overhead for the distribution network operator associated with coordinating and dealing with large numbers of small participants. Digital intermediation, enabled by automated algorithms running across integrated technologies, offers the potential for radical change in traditional centralised energy systems. The evolution of the role of the passive distribution network operator to that of the Distribution System Operator (DSO) [1] will bring a widespread change in the relationships of flexible generation and customers. These trends could place the aggregation of end users at the centre of the energy system.

Flexibility associated with distributed energy resources (DERs) has the potential to be aggregated by suppliers and

Funded through the Engineering and Physical Sciences Research Council (EPSRC) Aggregators as diGital Intermediaries in Local Electricity markets (AGILE) project (EP/S003088/1) third party aggregators to provide revenue streams in distribution and national level markets. Managed correctly, DER flexibility can defer network investments to meet increasing demand, by providing flexibility at peak times or support local system balancing. In contrast, current operational practice on distribution networks would encounter DER connection capacity being restricted or if significant, network reinforcement being invoked.

To ensure DER capacities will not be restricted, while respecting voltage and thermal constraints at distribution level, DER demand or generation set points could be validated dynamically, based on 3-phase low voltage network constraints. In this work, these constraints are analysed via load flow to model operational distribution feeders. A distribution level congestion management heuristic is proposed to maintain current or voltage within safe margins. The heuristic is suitable for DSO and aggregator planning use cases: network congestion management and market positions respectively. This methodology is part of a proposed digital aggregator platform which includes:

1) Agent based optimisation of DER 'agents'

2) A 3-phase network constraint management heuristic

3) Multi-market decision making tool for the aggregator portfolio

While this paper is focused on the network constraint management heuristic, the interaction with the other layers in the digital aggregator platform are also considered. The remainder of this paper is organised as follows: Section II contains a literature review of the state of the art in aggregator platforms and congestion management, Section III outlines the proposed digital aggregator platform and Section IV presents conclusions and recommendations for future work.

\section{LITERATURE REVIEW}

The literature on aggregators, or virtual power plants (VPPs) as they are sometimes referred, is extensive. There are numerous papers considering bidding behaviour of VPPs, particularly focusing on uncertainty in the VPP portfolio market position when including intermittent renewables [2]-[4]. However, such papers focus on optimisation of the VPPs portfolio in wholesale markets, and do not account for distribution system constraints which could affect the availability of DERs in a 
VPPs portfolio. Furthermore, most works on VPPs focus on larger generators, whereas this work extends the VPP literature by considering aggregation of larger numbers of domestic flexibility providers, or agents, such as EVs. In [5], the VPP provides a congestion management service to the DSO in a similar approach to the flexibility dispatch heuristic proposed in this work. This paper extends the work above by considering 3-phase unbalanced low voltage networks, whereas the above papers focus on single phase balanced network modelling at higher voltages.

\section{A. Aggregator platforms}

Although the concept of aggregator or VPP was conceived more than ten years ago, there is no uniform framework definition for implementation. The theory of an aggregator as an agent was discussed in [6] where a decentralised energy trading architecture focusing on an EV fleet was proposed to facilitate ancillary services. This was done by implementing $\mathrm{V} 2 \mathrm{G}$ in an agent-based model using Dutch smart metering data. The algorithm enabled local energy transfers between households and EVs with the aggregator being responsible for execution of the trading algorithm.

In [7] a flexibility framework was proposed to enable multiple participants to compete for selling or buying flexibility. Within the framework, an aggregator is defined as a local market operator that supervises flexibility transactions of the local energy community. Flexibility stakeholders that communicate with the aggregator are the DSO, the balance responsible party (that, in turn, has commitments in wholesale markets) and DER owners.

In a broad sense, aggregator coordination strategies can be divided into two control schemes: fully dispatchable (direct) and price driven (indirect).

- Fully dispatchable: suggests bidirectional aggregator-toprosumer communication where DERs are under the direct control of an aggregator or other type of VPP operator, e.g., a peer-to-peer trading platform as described in [8]. Such an approach provides responses at fast timescale, e.g. Firm Frequency Response (FFR).

- Price driven: suggests incentive signals to DER owners [9], where prosumers are expected to make generation decisions according to their individual preferences. Location based pricing and day ahead hourly pricing have been proposed to financially motivate DER owners to shift loads from peak demand periods and address potential congestion of distribution network. Consumer responsiveness to such time-varying prices was investigated in [10].

\section{B. Constraint management}

As networks operation approaches thermal and voltage limits, the DSO is increasingly being called on to actively manage network congestion, as an alternative to costly network reinforcement. Recent EU funded academic projects to develop models for the DSO as active system operator, include: SmartNet [11], Ecogrid 2.0 [12], [13] and INVADE [1]. Furthermore, the Universal Smart Energy Framework
(USEF) [14], which is being implemented in an industry DSO flexibility market trial [15], provides a detailed framework for DSO flexibility markets with the aggregator as the central market actor. However, guidance on determining congestion zones, and methods of assessing the probability of such congestion occurring, are sparse. The USEF framework defines a congestion point, a part of the grid where capacity might be exceeded, but leaves how to determine these congestion points up to the DSO.

Multiple methods exist to manage network congestion, and to represent this congestion with price signals, including: Distribution locational marginal prices [16], [17], multi-agent systems [18], [19] and the traffic light style implementation [20], [21]. However, examples of 3-phase methods applied to low voltage networks, where domestic EVs, PV and heatpumps will be located, are harder to come by. In [22], EV demands are adjusted to reduce the probability of congestion (thermal or voltage) in a line/node to a given value (e.g. $10 \%$ ). The networks are assumed to be balanced, whereas here unbalanced LV networks are modelled.

\section{Digital Aggregator Platform}

The digital aggregator platform illustrated in Figure 1 provides a link between: the aggregator, which has contracts with multiple agents including owners of EVs, PV, heat pumps and batteries at domestic scale, as well as larger flexible demands such as heating, ventilation and air conditioning (HVAC), refrigeration and combined heat and power (CHP) at larger scale; the network, on which the 'agents' are physically located, operated and monitored by the DSO; and the markets which could include national wholesale, frequency response, balancing and ancillary service markets.

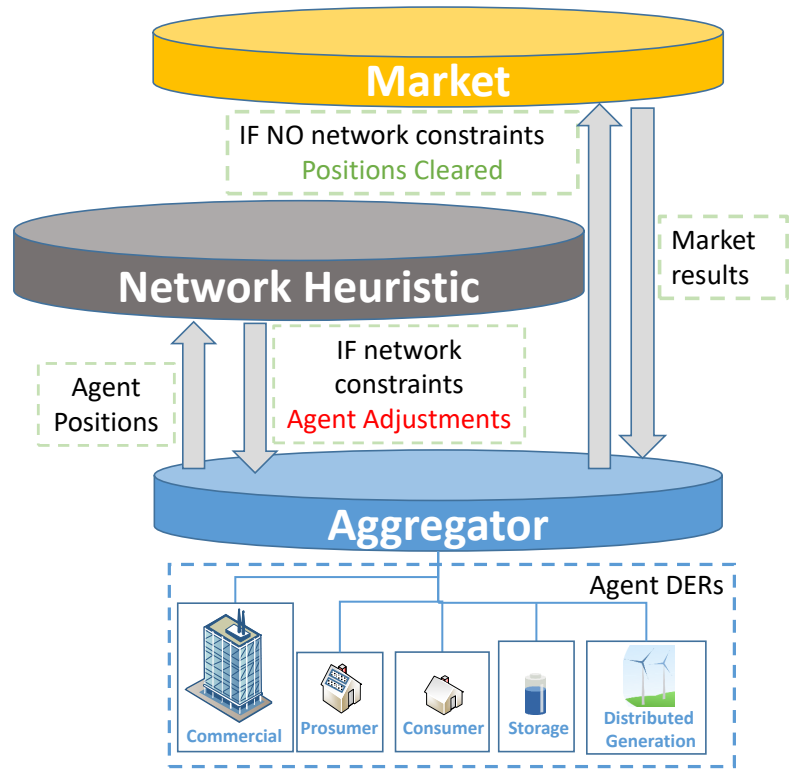

Fig. 1: Proposed digital aggregator platform layers.

The main function of the network heuristic is to ensure that actions by the aggregator in the markets, involving the 
adjustment of multiple DERs, do not result in thermal and voltage violations in the distribution network. Table I shows the main information exchanges between the layers in the digital aggregator platform: the aggregator passes agent positions to the network heuristic layer, once positions have been cleared they can be aggregated and passed to the market layer. The market layer will then pass market results to the aggregator such as contracts for availability or half-hourly cleared energy prices.

TABLE I: Information exchange between the layers of the aggregator platform.

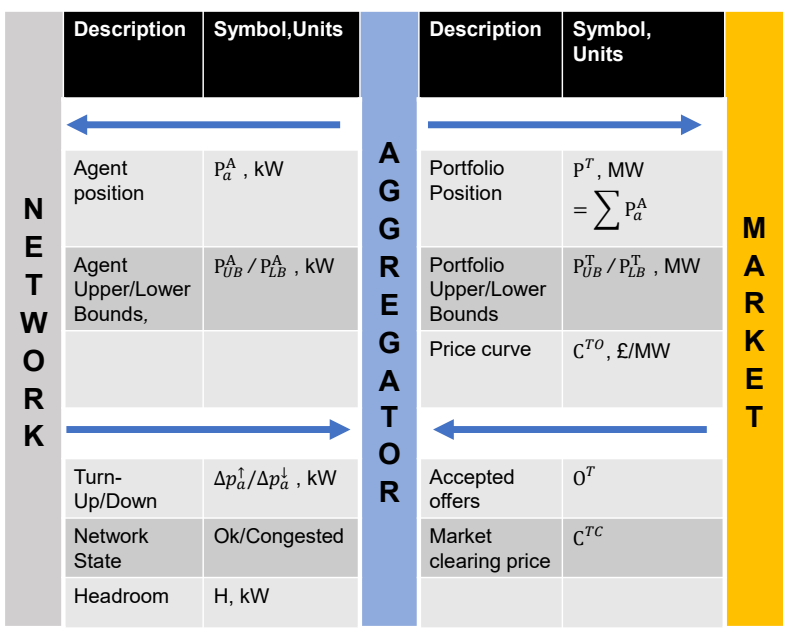

The stages of the aggregator portfolio optimisation are as follows;

1) Aggregator Multi Agent System used to optimise asset/agent portfolio. Agent positions are then passed to the network heuristic.

2) Network heuristic carries out load flow to check for thermal and voltage constraints.

a) IF no constraints: aggregator can submit offering to the national markets (e.g. wholesale day-ahead, intraday, balancing mechanism or ancillary services)

b) IF constraints: network heuristic carries out sensitivity analysis to determine agents most effective at solving constraints. Agent adjustments passed back to aggregator.

3) The aggregator can then optimise these adjustments based on agent pricing, repeating steps 1 and 2 if necessary.

4) Multi-market optimisation is carried out by the aggregator based on maximising profits or other objectives such as environmental improvement (e.g. decarbonisation) and community benefit (e.g. affordability) can also be optimised.

A Bayesian approach is to be applied to decision making, due to providing a means of propagating uncertainty associated with the network consequences of pricing signals. Variables will be encoded as probability distributions to articulate the plausible outcomes of dispatched load or generation, permitting the resulting measure of network congestion to also be represented as a probability distribution and therefore able to express the certainty with which particular outcomes will occur. The benefit of this is that actors in the system can then make decisions based on their attitude to risk by choosing specific quantiles of resulting probability distributions [23]. While this removes the challenge of detailed physical system modelling, it replaces it with a lesser challenge of correctly representing and combining relevant prior knowledge [24].

The aggregator multi-agent system and network heuristic layers of the digital aggregator platform are described in more detail in the following sections.

\section{A. Aggregator Multi-Agent System}

The aggregator multi-agent system facilitates DER portfolio optimisation. Power flow estimations are calculated for a 48 hour period using a distributed optimisation algorithm and passed to the network heuristic for feasibility verification. Each agent that represents a DER owner is characterised by flexible and inflexible components of load from the grid, with some DER owners having an EV, energy battery storage system or renewable energy source (or a combination of such assets).

1) EV simulation: To quantify uncertainties related to EVs' availability during delivery time periods, a discrete-time Markov chain with time-varying transition matrix is used for this study. The conditional probability of an EV going from the state $i$ at the moment $t-1$ to the state $j$ at the moment $t$ is given by:

$$
\pi_{i \rightarrow j}=P\left(z_{t}=j \mid z_{t-1}=i\right)
$$

The period of one simulation cycle is 48 hours with a 30 minute time step. A total of 96 timesteps is considered, and to be consistent with the terminology, $t=0$ is the initial moment and $z_{0}$ is the initial state. Three EV states are considered, $S=$ $\{M, R, C\}$, where $M$ is the movement state and $R$ and $C$ are the states indicating that an $\mathrm{EV}$ is parked in either residential or commercial area correspondingly. The transition matrix at the moment $t$ is given by:

$$
T_{t}=\left(\begin{array}{ccc}
\pi_{M \rightarrow M}^{t} & \pi_{M \rightarrow R}^{t} & \pi_{M \rightarrow C}^{t} \\
\pi_{R \rightarrow M}^{t} & \pi_{R \rightarrow R}^{t} & \pi_{R \rightarrow C}^{t} \\
\pi_{C \rightarrow M}^{t} & \pi_{C \rightarrow R}^{t} & \pi_{C \rightarrow C}^{t}
\end{array}\right)
$$

2) Commercial retail refrigeration loads: Commercial retail refrigeration loads are presented in the model in the form of multiple compressor pack systems as described in [25]. Each compressor pack is a fixed volume displacement device capable of providing flexibility via dynamic control of the pressure in the system and defrost re-scheduling for display cases. For fast scale responses, additional defrosts are initiated to provide a rapid reduction in power consumption. These defrosts self-terminate if the calculated product temperature $(\mathrm{CPT})$ reaches a pre-defined upper limit. $\mathrm{CPT}$ is calculated as follows: 


$$
\mathrm{CPT}_{k}=\frac{1}{30}\left(\sum_{i=1}^{29} \mathrm{CPT}_{k-i}+T_{\text {shelf }}^{k}\right)
$$

where $T_{\text {shelf }}^{k}$ is the shelf temperature at the $k$-th minute.

3) Linear state-of-charge model for battery assets: A linear state-of-charge $(\mathrm{SoC})$ model is used to describe the battery energy capacity and define its constraints over the simulation period. For the $i$-th prosumer, the constraints of the $j$-th battery at the charging rate $B_{j i}$ are given by:

$$
\operatorname{SoC}_{j i}^{\min } \leqslant \operatorname{SoC}_{j i}^{0}+E_{j i}^{-1} \Delta T \sum_{t=1}^{s} B_{j i}^{t} \leqslant \operatorname{SoC}_{j i}^{\max }, \forall s \in[1,96]
$$

where $\Delta T$ is the time interval, $E_{j i}$ is the battery energy capacity.

\section{B. Network heuristic}

The network heuristic considered in this work (Figure 2) takes inputs, including demand and generation at a given timestep, calculates the resulting voltages and currents on the network, and determines if they are within operating limits. Lower and upper voltage limits are assumed to be 0.94 and 1.1 p.u. respectively, in line with recommended operating standards in the UK [26]. In the event of thermal or voltage violations the following 3-phase sensitivity is carried out.

1) For phase $n$ (of 3):

a) if voltage or thermal violation, $V_{m}$ :

i) For agent a $\in \mathcal{A}$ :

A) Turn up/down active power, $\mathrm{P}_{a}^{\mathrm{A}}$, by $10 \%$, $=\Delta \mathrm{P}_{a}^{\mathrm{A}}$

B) Carry out load flow. Sensitivity, $\mathrm{S}_{a}^{\mathrm{A}}=$ $\Delta V_{m} / \Delta \mathrm{P}_{a}^{\mathrm{A}}$

ii) Order agents by highest sensitivity. And return feedback, $\mathrm{S}_{a} \in \mathcal{A}$ to aggregator.

The proposed method has the advantage of considering each phase individually, therefore only considering agents which have the largest impact on reducing violations when they occur on a specific phase. The heuristic can also be extended to calculate agent adjustments to solve constraints. Using probabilistic distribution of generation (PV) and demand (EV, Heat Pumps, baseload and commercial loads) it is proposed to use the heuristic to return the probability of agent adjustments. The aggregator can then plan their commitment to national markets based on the probability that distribution network congestion could reduce the flexibility available from the agents.

\section{CONCLusions And Future Work}

Methods of managing distribution network constraints and the uncertainty around flexibility from multiple smaller DERs or 'agents', such as EVs, will be crucial to decentralisation of the electricity system. This paper identifies that the adoption of a congestion management strategy for aggregating flexibility from agents to the DSO and national markets is a necessary component of current aspirations in system operation. A digital aggregator platform which uses a heuristic management strategy is outlined. Such an approach is considered valuable in determining new systems operation functions - for example, the timely calculation of congestion probabilities, and in recommended agent adjustments to resolve this congestion. In future work, probabilistic methods or empirical data could be used to sample distributions of demand, generation and flexibility, and using these input distributions with the network congestion management heuristic, produce output probabilities for each agent, giving customised recommended adjustments due to congestion. The digital aggregator can then combine the flexibility from multiple agents, using probabilities for the aggregated portfolio to assess the risk to delivery in a multimarket decision optimisation.

\section{REFERENCES}

[1] Energy Networks Association, "Open Networks Project Consultation on future worlds," Tech. Rep. March, 2019.

[2] M. Peik-Herfeh, H. Seifi, and M. Sheikh-El-Eslami, "Decision making of a virtual power plant under uncertainties for bidding in a day-ahead market using point estimate method," International Journal of Electrical Power \& Energy Systems, vol. 44, no. 1 , pp. 88 - 98, 2013. [Online]. Available: http://www.sciencedirect.com/science/article/pii/S0142061512003766

[3] J. Z. Riveros, K. Bruninx, K. Poncelet, and W. D'haeseleer, "Bidding strategies for virtual power plants considering chps and intermittent renewables," Energy Conversion and Management, vol. 103, pp. $408-418,2015 . \quad$ [Online]. Available: http://www.sciencedirect.com/science/article/pii/S0196890415006238

[4] A. G. Zamani, A. Zakariazadeh, S. Jadid, and A. Kazemi, "Stochastic operational scheduling of distributed energy resources in a large scale virtual power plant," International Journal of Electrical Power \& Energy Systems, vol. 82, pp. 608 - 620, 2016. [Online]. Available: http://www.sciencedirect.com/science/article/pii/S0142061516306615

[5] D. Koraki, J. Keukert, and K. Strunz, "Congestion management through coordination of distribution system operator and a virtual power plant," in 2017 IEEE Manchester PowerTech, June 2017, pp. 1-6.

[6] S. Hijgenaar, Z. Erkin, T. Keviczky, J. Siemons, R. Bisschops, and A. Verbraeck, "A decentralised energy trading architecture for future smart grid load balancing," in 2017 IEEE International Conference on Smart Grid Communications (SmartGridComm). IEEE, Oct. 2017. [Online]. Available: https://doi.org/10.1109/smartgridcomm.2017.8340707

[7] P. Olivella-Rosell, P. Lloret-Gallego, Í. Munné-Collado, R. VillafafilaRobles, A. Sumper, S. Ottessen, J. Rajasekharan, and B. Bremdal, "Local flexibility market design for aggregators providing multiple flexibility services at distribution network level," Energies, vol. 11, no. 4, p. 822, Apr. 2018. [Online]. Available: https://doi.org/10.3390/en11040822

[8] T. Morstyn, N. Farrell, S. J. Darby, and M. D. McCulloch, "Using peer-to-peer energy-trading platforms to incentivize prosumers to form federated power plants," Nature Energy, vol. 3, no. 2, pp. 94-101, Feb. 2018. [Online]. Available: https://doi.org/10.1038/s41560-017-0075-y

[9] K. Heussen, S. You, B. Biegel, L. H. Hansen, and K. B. Andersen, "Indirect control for demand side management - a conceptual introduction," in 2012 3rd IEEE PES Innovative Smart Grid Technologies Europe (ISGT Europe). IEEE, Oct. 2012. [Online]. Available: https://doi.org/10.1109/isgteurope.2012.6465858

[10] J. Schofield, R. Carmichael, S. Tindemans, M. Woolf, M. Bilton, and G. Strbac, "Residential consumer responsiveness to time-varying pricing," 2014.

[11] G. Leclercq, M. Pavesi, T. Gueuning, A. Ashouri, P. Sels, F. Geth, D. Reinhilde, and H. Le Cadre, "SmartNet D2.2 Network and Market Models," Tech. Rep. D2.2, 2018.

[12] C. Kok, J. Kazempour, and P. Pinson, "A DSO-Level Contract Market for Conditional Demand Response," pp. 1-6, 2019. 


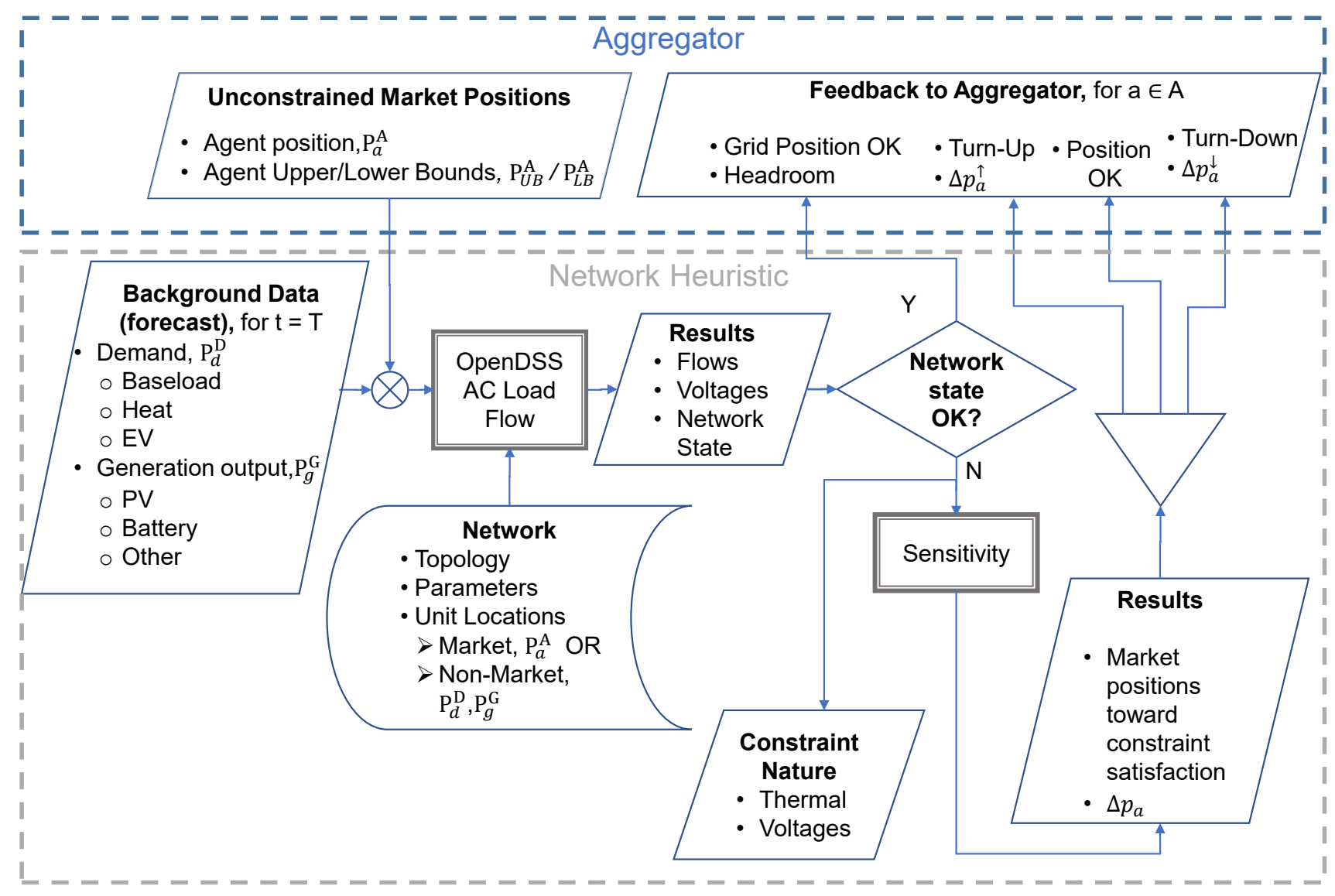

Fig. 2: Proposed network congestion management heuristic

[13] L. Bobo, S. Delikaraoglou, N. Vespermann, J. Kazempour, and P. Pinson, "Offering strategy of a flexibility aggregator in a balancing market using asymmetric block offers," 20th Power Systems Computation Conference, PSCC 2018, vol. 0, pp. 1-7, 2018.

[14] USEF Foundation, USEF: The Framework Specifications, 2015 [Online]. Available: https://www.usef.energy/download-the-framework/

[15] SP Energy Networks, "Network Innovation Competition Proposal: FUSION," 2017. [Online]. Available: http://www.ecogrid.dk/en/home uk\#new-downloads

[16] J. Zhao, Y. Wang, G. Song, P. Li, C. Wang, and J. Wu, "Congestion Management Method of Low-Voltage Active Distribution Networks Based on Distribution Locational Marginal Price," IEEE Access, vol. 7, pp. 32 240-32 255, 2019.

[17] B. Biegel, P. Andersen, J. Stoustrup, and J. Bendtsen, Congestion management in a smart grid via shadow prices. IFAC, 2012, vol. 8, no. PART 1. [Online]. Available: http://dx.doi.org/10.3182/20120902-4FR-2032.00091

[18] T. H. Vo, A. N. Haque, P. H. Nguyen, I. G. Kamphuis, M. Eijgelaar, and I. Bouwman, "A study of congestion management in smart distribution networks based on demand flexibility," 2017 IEEE Manchester PowerTech, Powertech 2017, 2017.

[19] J. A. Greunsven, E. Veldman, P. H. Nguyen, J. G. Slootweg, and I. G. Kamphuis, "Capacity management within a multi-agent marketbased active distribution network," IEEE PES Innovative Smart Grid Technologies Conference Europe, pp. 1-8, 2012.

[20] S. Y. Hadush and L. Meeus, "DSO-TSO cooperation issues and solutions for distribution grid congestion management," Energy Policy, vol. 120, no. June, pp. 610-621, 2018.

[21] R. Villafafila-Robles, B. Bremdal, A. Sumper, J. Rajasekharan, P. Olivella-Rosell, S. Ottessen, Í. Munné-Collado, and P. Lloret-Gallego, "Local Flexibility Market Design for Aggregators Providing Multiple Flexibility Services at Distribution Network Level," Energies, vol. 11, no. 4 , p. 822,2018
[22] J. Romero-Ruiz, J. Pérez-Ruiz, S. Martin, J. Aguado, and S. D. la Torre, "Probabilistic congestion management using evs in a smart grid with intermittent renewable generation," Electric Power Systems Research, vol. 137, pp. 155 - 162, 2016. [Online]. Available: http://www.sciencedirect.com/science/article/pii/S0378779616300621

[23] Jun Zhang, Jian Pu, J. D. McCalley, H. Stern, and W. A. Gallus, "A bayesian approach for short-term transmission line thermal overload risk assessment," IEEE Transactions on Power Delivery, vol. 17, no. 3, pp. 770-778, July 2002.

[24] P. Parpart, M. Jones, and B. C. Love, "Heuristics as bayesian inference under extreme priors," Cognitive Psychology, vol. 102, pp. $127-144,2018 . \quad$ [Online]. Available: http://www.sciencedirect.com/science/article/pii/S0010028517303286

[25] A. Postnikov, I. Albayati, S. Pearson, C. Bingham, R. Bickerton, and A. Zolotas, "Facilitating static firm frequency response with aggregated networks of commercial food refrigeration systems," Applied Energy, vol. 251, p. 113357, Oct. 2019. [Online]. Available: https://doi.org/10.1016/j.apenergy.2019.113357

[26] Energy Networks Association, "Engineering Recommendation P2/6 Security of Supply," Tech. Rep., 2006. 